\title{
THE TEMPERATURE SCALE FOR CLASSICAL CEPHEIDS
}

\author{
SIDNEY B. PARSONS
}

Dept. of Astronomy, The University of Texas at Austin, Tex., U.S.A.

\begin{abstract}
In the study of Cepheid pulsation and evolution, it is very important to be able to obtain effective temperatures from some easily observable spectral characteristic. A review is given of the several discrepant relations between $T_{\text {eff }}$ and intrinsic $B-V$ index given for classical Cepheids and/or yellow supergiants by Rodgers (1970) and Parsons (1971) (the same as Kraft, 1961; Böhm-Vitense, 1972; Schmidt, 1972, and van Paradijs, 1973). All temperatures agree well at $(B-V)_{0}=0.4$ but diverge toward redder colors; at $(B-V)_{0}=0.9$ the values range from $4900^{\circ}(B o ̈ h m-V i t e n s e$, for class Ia-Ib) to $5770^{\circ}$ (Schmidt, for class I stars). In each case the analysis leading to the assignment of $T_{\text {eff }}$ to a given color index is not completely satisfactory, so it is difficult to make value judgements. Comparison of relations between $T_{\text {eff }}$ and spectral class shows similar discrepancies.
\end{abstract}

The author's 1971 analysis of UVBGRI photometry of 101 supergiants and Cepheids was based on a single-valued relation between line-blocking in each wavelength interval and $T_{\text {eff }}$. The slope of the $T_{\text {eff }}$ vs $(B-V)_{0}$ relation was determined à la Oke (1961) by maximizing agreement between the photometric and spectroscopic radius variations in two Cepheids, while the absolute scale was fixed from the F5 Ib star $\alpha$ Per. The adopted value of $6425^{\circ}$ (with $E(B-V) \simeq 0.06$ ) is supported by recent unpublished calculations of $\mathrm{H} \beta, \mathrm{H} \gamma$, and $\mathrm{H} \delta$ profiles which fit the observed profiles in $\alpha$ Per quite well (except within $0.3 \AA$ of line center) for $T_{\text {eff }}=6350 \pm 100$. For stars much cooler than this, though, the Balmer line wings are not strong enough to serve as accurate criteria.

Analysis of continuous energy distributions is hampered by line-blocking corrections and by interstellar reddening. From synthetic spectra computed from model atmospheres in collaboration with R. A. Bell, a good 'reddening-free' parameter was found for the Stebbins and Kron system:

$$
Q_{1}=(V-G)-0.86(G-I) \text {. }
$$

The relation between this parameter and intrinsic Violet-Green index is nearly independent of gravity, abundance and microturbulence. In fact, it is found empirically that the Cepheids follow a single curve in the $V-G$ vs $Q_{1}$ diagram during their pulsation, with no evidence for looping except in the case of $l$ Car. The shift in $V-G$, for a given Cepheid, to the intrinsic relation from the model atmospheres yields the absolute color excess, independent of the cluster method. The resulting $E(B-V)$ values confirm Parsons' 1971 results and average about 0.07 mag. smaller than other values in the literature.

The determination of reddening does not solve the $T_{\text {eff }}$ problem, but if there is a unique temperature scale for Cepheids and if a given star is 0.07 mag. redder than 
previously thought, then that star is about $150^{\circ}$ cooler. This goes part way toward removing the discrepancy between evolutionary and pulsational masses, even keeping the $T_{\text {eff }}$ vs $(B-V)_{0}$ relation fixed. At present there are no strong arguments against continuing to use the Oke-Kraft-Parsons relation

$$
\log T_{\text {eff }}=3.886-0.175(B-V)_{0},
$$

which is not significantly different from Rodgers' relation and is intermediate between the extremes. However, one must recognize that not only the equation but also the inputs are subject to some uncertainty, and that the use of $\langle B\rangle-\langle V\rangle$ instead of $\langle B-V\rangle$ does not give a meaningful value of $\left\langle\log T_{\text {eff }}\right\rangle$.

\section{References}

Böhm-Vitense, E.: 1972, Astron. Astrophys. 17, 335.

Kraft, R. P.: 1961, Astrophys. J. 134, 616.

Oke, J. B.: 1961, Astrophys. J. 134, 214.

Paradijs, J. van: 1973, Astron. Astrophys. 23, 369.

Parsons, S. B.: 1971, Monthly Notices Roy. Astron. Soc. 152, 121.

Rodgers, A. W.: 1970, Monthly Notices Roy. Astron. Soc. 151, 133.

Schmidt, E. G. : 1972, Astrophys. J. 174, 605.

\section{DISCUSSION}

McNamara: As you're aware, the various determinations of colour excess wander all over the place in a systematic fashion, yet a student by the name of Kent Phelps who's determining colour excesses from field stars in the vicinity of Cepheids, very carefully going round doing photometry, has determined the colour excesses now for about 7 or 8 stars. His colour excesses were compared to various observers and you'll be glad to hear that they agree with yours better than anybody else. But still they are systematically different from yours again by about two hundredths of a magnitude in $(b-y)$. The colour excesses are generally, I think he would conclude, overestimated; and yours also slightly overestimated.

Rodgers: I'd like to ask you what do you think of Schmidt who determined a temperature scale from fitting deblanketed $(R-I)$ to your models and put the temperature back up again. He makes the point in the paper, which I could find difficulty in accepting, that the increased accuracy in determining the blanketing of $R$ and $I$ makes the method superior to one dependent on $(B-V)$. The actual colour difference in $(R-I)$ is so small for stars of $6000^{\circ}$ that observational errors become significant, it seems to me.

Parsons: I haven't seen this unfortunately but I'm sure $(R-I)$ should be a good criterion. That part of the spectrum was certainly included in the six colour fitting.

Schatzman: Are your models based on stationary atmospheres with constant gravity and temperature? Does it include a possibility of velocity gradient, passage of shock fronts, something like that in the atmosphere?

Parsons: No, it does not. They're completely static.

Iben: One drawback of changing the temperature scale is that if you fit in a mass-luminosity diagram, you leave a gap in the H-R diagram between the blue edges and the Cepheids, which would be as embarassing as a mass discrepancy.

Cox: But then you could vary the helium content. (laughter)

Parsons: Even by a change of 7 hundredths of a magnitude in $E(B-V)$, one does not probably change, move the Hyades. When Sandage and Tammann derived their P-L-C relationship, they used a ratio of total to selective absorption of 3.0 which is too low for these cooler stars. 